\title{
Estomaterapeutas no mundo do trabalho: facilidades e dificuldades para o exercício profissional
}

\author{
Stomatherapists in the world of work: Practicalities and difficulties for the professional practice \\ Estomaterapeutas en el mundo del trabajo: facilidades y dificultades para el ejercicio
}

Carolina Cabral Pereira da $\operatorname{Costa}^{1}$ (1) Samira Silva Santos Soares ${ }^{2}$ (1) Manoel Luís Cardoso Vieira ${ }^{3}$ (I) Midian Dias Oliveira ${ }^{1}$ (i) Raquel Soares Pedro ${ }^{1}$ (D) Ursula Silva Baptista Chaves ${ }^{4}$ (C) Norma Valéria Dantas de Oliveira Souza ${ }^{1}$ [C]

1.Universidade do Estado do Rio de Janeiro, Faculdade de Enfermagem. Rio de Janeiro, RJ, Brasil.

2. Universidade Federal do Rio de Janeiro, Escola de Enfermagem Anna Nery. Rio de Janeiro, RJ, Brasil.

3. Universidade Federal do Rio de Janeiro, Instituto de Doenças do Tórax. Rio de Janeiro, RJ, Brasil.

4. Centro Universitário Celso Lisboa. Rio de Janeiro, RJ, Brasil.

AUTOR CORRESPONDENTE:

Carolina Cabral Pereira da Costa

E-mail: carolcuerj@hotmail.com

Recebido em 10/07/2020.

Aprovado em 10/10/2020.

DOl:https://doi.org/10.1590/2177-9465-EAN-2020-0262

\section{RESUMO}

Objetivo: analisar as facilidades e dificuldades percebidas por egressos de uma pós-graduação em Estomaterapia para atuação no mundo do trabalho. Métodos: pesquisa qualitativa, desenvolvida com 22 egressos de um Curso de Pós-graduação em Enfermagem em Estomaterapia, de uma universidade pública da Região Sudeste do Brasil. A coleta de dados ocorreu por meio de entrevista semiestruturada, entre janeiro e abril de 2018. As entrevistas foram tratadas com base na análise temática de conteúdo. Resultados: apreenderam-se como facilidades: reconhecimento do especialista, autonomia profissional, conhecimentos adquiridos por meio da especialização, disponibilidade de tecnologias de cuidados. Como fatores dificultadores, citaram-se: carência de recursos humanos e materiais, baixa remuneração do especialista, política institucional desfavorável, não valorização do estomaterapeuta. Conclusões e implicações para a prática: as facilidades identificadas se relacionaram com o reconhecimento do especialista; a autonomia; a ascensão e o crescimento profissional; os conhecimentos adquiridos por meio da especialização; e a disponibilidade de tecnologias do cuidado para realização da prática profissional. As dificuldades analisadas no estudo estiveram vinculadas predominantemente à configuração do mundo do trabalho, cujos fundamentos se pautam no ideário neoliberal do Estado mínimo e no enxugamento da máquina pública.

Palavras-chave: Mercado de trabalho; Enfermagem; Especialidades de enfermagem; Papel profissional; Trabalho.

\section{ABSTRACT}

Objective: to analyze the facilities and difficulties perceived by graduates of a Post-Graduation in Stomatherapy to work in the world of work. Methods: qualitative research, developed with 22 graduates from a Postgraduate course in Nursing in Stomatherapy, from a public university in the Southeast of Brazil. Data collection took place through semi-structured interviews, between January and April 2018. The interviews were treated based on the Thematic Content Analysis. Results: it was apprehended as facilities: recognition of the specialist, professional autonomy, knowledge acquired through specialization, availability of care technologies. As hindering factors, the following were cited: lack of human and material resources, low remuneration of the specialist, unfavorable institutional policy, lack of appreciation for the stomatherapist. Conclusion and implication for the practice: the difficulties analyzed in the study were predominantly linked to the configuration of the world of work, whose foundations are based on the neoliberal ideas of the minimum state and the wiping out of the public machinery.

Keywords: Job Market; Nursing; Specialties nursing; Professional role; Work.

\section{RESUMEN}

Objetivo: analizar las instalaciones y dificultades percibidas por los egresados de una Postgrado en Estomatoterapia para trabajar en el mundo laboral. Métodos: investigación cualitativa, desarrollada con 22 graduados de un curso de Postgrado en Enfermería en Estomatoterapia, de una universidad pública en la Región Sudeste de Brasil. La recopilación de datos se realizó a través de entrevistas semiestructuradas, entre enero y abril de 2018. Las entrevistas se trataron en función del análisis de contenido temático. Resultados: fue aprehendido como instalaciones: reconocimiento del especialista, autonomía profesional, conocimiento adquirido a través de la especialización, disponibilidad de tecnologías de atención. Como factores que obstaculizan, se citaron los siguientes: falta de recursos humanos y materiales, baja remuneración del especialista, política institucional desfavorable, falta de valoración del terapeuta del estoma. Conclusión e implicación para la práctica: las dificultades analizadas en el estudio estuvieron principalmente relacionadas con la configuración del mundo del trabajo, cuyos fundamentos se basan en las ideas neoliberales del estado mínimo y la eliminación de la maquinaria pública.

Palabras clave: Mercado de trabajo; Enfermería; Especialidades de enfermería; Rol profesional; Trabajo. 


\section{INTRODUÇÃO}

O objeto deste estudo trata das facilidades e dificuldades percebidas por egressos de um Curso de Estomaterapia, da Região Sudeste do Brasil, para atuarem como especialistas no mundo do trabalho. A temática emergiu a partir de observações empíricas, captadas em conversas informais com especialistas da área, que expressavam sentimentos dialéticos quanto à atuação no mercado de trabalho, ora salientando prazer, ora referindo sofrimento. A manifestação de tais sentimentos parecia envolver situações diversas, como a configuração da organização laboral, a conformação político-econômica do trabalho, o reconhecimento e a valorização da enfermagem e da estomaterapia.

A Pós-graduação Lato sensu em Enfermagem é tida como uma das atuais possibilidades de qualificar enfermeiros para a prática profissional, contribuindo para a transformação e excelência da enfermagem. Nesse contexto, tem-se a especialidade Enfermagem em Estomaterapia, a qual surgiu no final da década de 1950, nos Estados Unidos da América, e, desde então, difundiu-se globalmente. ${ }^{1,2}$

O enfermeiro pós-graduado em Estomaterapia é denominado estomaterapeuta, sendo aquele que possui conhecimento e habilidades para cuidar de pessoas com estomias, feridas, fístulas, cateteres, drenos e incontinência anal e urinária. Este especialista atua nos campos do ensino, da assistência, da pesquisa, da administração, das vendas, da assessoria e consultoria. ${ }^{2}$ A especialidade vem crescendo no Brasil, e muitos enfermeiros têm buscado a qualificação nesta área, com objetivo de adquirir expertise e ampliar as possibilidades de atuação.

Salienta-se que o atual mercado de trabalho é marcado por elevadas exigências, fruto de significativas transformações mundiais decorrentes do desenvolvimento científico, econômico, tecnológico, motivando os profissionais a se adequarem rapidamente às novas tendências impostas pelo mundo laboral. Assim, características e requisitos se fazem necessários aos profissionais, como iniciativa, autonomia, capacidade de resolução de problemas, criatividade, dentre outros, para conseguirem inserção e manutenção nesse mercado. . $^{3-5}$

Enfatiza-se, também, que o modelo econômico predominante no Brasil se fundamenta no ideário neoliberal, o que, entre outras repercussões para o mundo do trabalho, resulta em elevadas exigências para os trabalhadores mais qualificados, ressaltandose habilidades como criatividade, flexibilidade e capacidade de inovar, conhecimento específico e resiliência para lidar com a pressão e o ritmo acelerado das mudanças. ${ }^{3-5}$

Ademais, em decorrência de preceitos neoliberais, verifica-se o enxugamento da máquina pública, acarretando a precarização das condições de trabalho. Outrossim, constatam-se a diminuição de concursos públicos e postos de trabalho para a enfermagem, ocasionando medo de perder o emprego, competitividade entre pares e baixa solidariedade no contexto laboral. ${ }^{4,5}$

É fundamental que o futuro especialista, ainda durante o processo de qualificação, vislumbre, por meio de atividades teórico-práticas, as facilidades e/ou dificuldades que poderão encontrar na realidade laboral. Isto é importante para que o profissional tenha, na dinâmica de trabalho, maior empoderamento e tomadas de decisões acertadas, possibilitando cuidado diferenciado e efetivo.

É igualmente necessário refletir sobre a necessidade de convergência entre os interesses do processo de ensinoaprendizagem e a infraestrutura material dos serviços de saúde, no intuito não apenas de formar estomaterapeutas que possam atuar com qualidade diante das limitações impostas no trabalho em saúde, mas de fortalecer a capacidade crítica e política desses profissionais, a fim de reivindicar melhores condições de trabalho. Desta maneira, contribui-se para o sentimento de prazer e reconhecimento pelo labor desenvolvido.

Em vista da problemática pontuada, objetivou-se analisar as facilidades e dificuldades percebidas por egressos de um Curso de Pós-graduação em Estomaterapia para atuação no mundo do trabalho.

\section{MÉTODOS}

Estudo de natureza qualitativa, do tipo descritivo-exploratório, realizado com egressos de um Curso de Pós-graduação em Enfermagem em Estomaterapia, de uma universidade pública da Região Sudeste do Brasil. Este curso atende às normativas recomendadas pela Associação Brasileira de Estomaterapia e pelo World Council of Enterostomal Therapists, tem duração de 14 meses, na modalidade presencial, englobando atividades teóricas, teórico-práticas e práticas. $O$ processo de seleção para o referido curso ocorre por meio de seleção pública, análise curricular e prova com questões objetivas, sendo aprovado o candidato com média igual ou superior a 7,0.

Elencou-se como critério de inclusão dos participantes: ser especialista em estomaterapia pela universidade mencionada com, no mínimo, três anos de qualificação. Tal critério temporal se baseou no fato de considerar esse tempo suficiente para os egressos terem se apropriado do que está relacionado com o exercício da especialidade. Ademais, o recorte temporal para inclusão se justifica porque se entende como recém-egressos aqueles formados até três anos, os quais ainda estão em processo de consolidação no mercado de trabalho. ${ }^{6}$

O critério de exclusão estabelecido foi ser egresso da turma de 2007, ano da primeira turma do curso, uma vez que, à época, não havia registros consistentes, na secretaria lato sensu, referentes aos dados para contato com os possíveis participantes dessa turma.

A partir dos critérios elencados, considerou-se o recorte temporal de seis anos, determinando-se como alvo populacional os egressos titulados entre 2008 e 2013. A coleta de dados ocorreu por meio de entrevista semiestruturada, realizada de janeiro a abril de 2018. Participaram do estudo 22 egressos do curso de pós-graduação mencionado, selecionados mediante a técnica da "bola de neve". Assim, escolheram-se, inicialmente, três egressos de turmas que se encontravam no critério temporal estabelecido. A estes, solicitou-se a indicação de outros egressos que estivessem incluídos nos critérios de seleção do estudo. As entrevistas foram interrompidas quando os participantes 
passaram a repetir os conteúdos obtidos anteriormente, atingindose o ponto de saturação.

As entrevistas foram realizadas e gravadas em local reservado e horário combinado, segundo a disponibilidade dos participantes, tendo duração média de 20 minutos. Houve transcrição na íntegra do conteúdo das entrevistas, no editor de texto Microsoft Word. Assim, pediu-se aos participantes que comentassem a respeito das facilidades e dificuldades para atuação como estomaterapeuta no atual mundo do trabalho em saúde. Isso deu margem para um diálogo dirigido para obtenção do objeto da pesquisa. Destaca-se que a pergunta foi elaborada pela equipe de pesquisadores deste estudo.

As entrevistas foram tratadas com base na técnica de análise temática de conteúdo, ${ }^{7}$ alcançando-se total de 746 Unidades de Registro(UR). Salienta-se que não houve a utilização de software para tratamento dos dados. Tal procedimento foi executado manualmente pela pesquisadora principal, empregando-se conhecimentos de matemática e estatística, possibilitando, desta maneira, contabilizar as UR e as unidades de significação.

A fim de manter o sigilo em relação à identificação dos participantes do estudo, adotou-se a letra E (Estomaterapeuta), seguida de número cardinal que representou a ordem cronológica de realização das entrevistas. De acordo com os padrões éticos exigidos, o estudo foi avaliado pelo Comitê de Ética em Pesquisa sendo aprovado conforme parecer n. 2.314.626/2017 e Certificado de Apresentação para Apreciação Ética n. 0107217.8.0000.5282.

\section{RESULTADOS}

A partir do tratamento dos dados, por meio da análise temática de conteúdo, emergiu a categoria denominada "Atuação no mundo do trabalho: facilidades e dificuldades percebidas pelos egressos". Esta categoria abarcou duas subcategorias, as quais foram intituladas: i) Facilidades vivenciadas pelos egressos no mundo do trabalho; e ii) Fatores dificultadores da atuação do especialista no mundo do trabalho.

\section{Atuação no mundo do trabalho: facilidades e dificuldades percebidas pelos egressos}

\section{Facilidades vivenciadas pelos egressos no mundo do trabalho}

A presente subcategoria apresentou 370 UR. Os participantes reconheceram que possuir o título de especialista em Estomaterapia concorreu significativamente para inserção e posterior manutenção no mundo do trabalho, com novas possibilidades de mercado.

Por eu ter a especialização, eu fui representante por cinco anos em uma multinacional e uma distribuidora de curativos. E eu queria trabalhar em comissão de curativo, eu nunca tive essa ambição, mas sabe quando as coisas vão acontecendo na sua vida? Os convites foram chegando naturalmente. Foi uma inserção bem rápida na área. (E7)
O fato de terem concluído o curso em uma universidade pública propiciou aos egressos maior reconhecimento social e profissional. A formação na referida instituição foi considerada fator impulsionador para inserção no mercado de trabalho.

O nome da instituição influencia sim, o nome tem peso, o nome te dá uma boa entrada nos lugares, maior reconhecimento, principalmente neste momento em que estamos vivendo de precarização dos postos de trabalho e dos salários. (E12)

O currículo do curso de especialização é um dos mais completos que tem no Brasil. Você tem as portas abertas com muita facilidade, é um diferencial. Além disso, ele é reconhecido pela Associação Brasileira de Estomaterapia (Sobest) e pela World Council of Enterostomal Therapists (WCET), o que também confere diferencial. Quando se fala o local de formação, abrem-se portas para trabalhar em diversas instituições, não só na assistência, como também na docência. (E20)

Outros aspectos sublinhados foram o reconhecimento e a valorização do trabalho executado pelo estomaterapeuta, entre os pares e pela sociedade.

Uma facilidade é que quando as pessoas veem o resultado do seu trabalho enquanto especialista, que aquilo que você propôs deu certo, você passa a ser respeitado, valorizado no seu ambiente de trabalho pelos demais profissionais. (E01)

A facilidade que eu tive foi com relação ao meu trabalho atual, de ser reconhecida como estomaterapeuta e ter sido chamada para trabalhar por conta disso, por conta dessa especialidade. É maravilhoso ver o reconhecimento do meu trabalho como estomaterapeuta dentro de uma área específica pelos meus pares. (E17)

Os egressos relataram, também, a possibilidade de atuar de forma autônoma.

A facilidade é que você tem autonomia para exercer essa função. Não preciso do arcabouço de uma instituição, seja ela pública ou privada, para que eu possa exercer essa especialidade. Eu posso simplesmente exercê-la, visitando a casa do paciente, por exemplo. (E06)

Verificou-se que um fator facilitador, possibilitado pela especialização, foi a oportunidade de ascensão na instituição em que esses especialistas trabalhavam, em decorrência da conclusão da pós-graduação.

Hoje, eu assumo um cargo que, com certeza, se eu não tivesse a especialização, eu não assumiria. Então, a especialização ajudou na minha ascensão profissional. 
O estomaterapeuta, no meu trabalho, não somente pode ser líder de equipe, como também no meu caso, fui convidada para ser presidente da comissão de curativos, de um grande hospital, que tem na equipe cirurgiões plásticos, médicos clínicos, fisioterapeutas. Deste modo, o presidente é quem traça as condutas, claro, após ouvir o grupo. (E20)

Ainda, comentaram a facilidade de, atualmente, as instituições disponibilizarem uma série de produtos/materiais para uso em pacientes, permitindo, assim, assistência de melhor qualidade.

Hoje em dia, a maioria dos hospitais tem variedade boa de material. Então, eu acho que isso é uma facilidade. $A$ gente tem, por exemplo, "hidrogel", "adaptic", diferente de antigamente que só tinha "colagenase" e, no máximo, "AGE". Na minha percepção, isso facilita nossa atuação, tornando-a mais resolutiva, de melhor qualidade. (E16)

\section{Fatores dificultadores da atuação do especialista no mundo do trabalho}

Esta subcategoria apresentou 376 UR. Embora os egressos tenham declarado como fator facilitador para atuação no mundo do trabalho o fato de as instituições apresentarem uma gama importante de materiais para a prática profissional, alguns estomaterapeutas entrevistados destacaram a carência desses materiais em instituições de saúde, sobretudo, nas públicas, como elemento que fragiliza o processo de trabalho desse especialista.

As dificuldades, elas, às vezes, são muito maiores. Por quê? Nós temos o conhecimento, só que nós não conseguimos, muitas vezes, empregar devidamente isso porque falta material. Nós não temos o que aprendemos durante a pós-graduação, na prática. Não temos os insumos, principalmente no serviço público. (E08)

Os egressos citaram como outra dificuldade o diminuto quantitativo de recursos humanos para viabilizar um cuidado efetivo e seguro.

A gente vivencia uma realidade de falta de pessoal. Além de ter pouco enfermeiro, faltam os especialistas nas instituições. (E01)

A gente trabalha com uma equipe em que a quantidade de enfermeiros é muito reduzida para o atendimento, inclusive para a quantidade de técnicos e auxiliares que a gente tem no mercado, então sobrecarrega. (E06)

Outra situação destacada pelos egressos foi o desconhecimento da população quanto à Estomaterapia, fator que dificulta a atuação do especialista nesta área.
A gente ainda é pouco conhecida enquanto especialidade. As pessoas pouco sabem o que é um estomaterapeuta e o que ele desenvolve, então, nossa atuação fica comprometida. (E14)

Além disso, constatou-se que a Estomaterapia possui campo restrito, configurando-se como dificuldade para atuação.

Então, a verdade é que você não tem muito campo de trabalho. São poucos locais que você tem a especialização disponível. A gente sabe que o campo de trabalho hoje em dia ainda é pequeno, é reduzido. Então, eu acho que é bem complicado. O campo no Rio de Janeiro está muito reduzido. (E16)

\section{DISCUSSÃO}

O mundo do trabalho tem sido pautado pelo ideário neoliberal, o qual não se caracteriza somente por uma filosofia econômica, mas também por um modo de viver que influencia valores culturais e emocionais, modificando a vida em sociedade e as relações de trabalho. ${ }^{4}$ Nesta perspectiva, a transformação do mundo do trabalho, suscitada pelo neoliberalismo, tem redefinido a configuração das organizações e dos processos laborais, que restringe alguns postos de trabalho, mas oferece novas frentes de atuação, especialmente para os mais qualificados e que detêm o conhecimento de tecnologias mais densas. ${ }^{3-5,8}$

Enfatiza-se que o mundo do trabalho contemporâneo vem sendo marcado pela globalização e modernização tecnológica, valorização do conhecimento qualificado e habilidade dos trabalhadores em resolver problemas. Portanto, a demanda de mercado tem impulsionado os profissionais a se capacitarem em cursos de especialização, mestrado e doutorado como estratégia para responderem às exigências e pressões laborais cada vez mais complexas e inovadoras. ${ }^{9}$

Nesse sentido, o mundo do trabalho tem colaborado para inserção dos estomaterapeutas no mercado, inclusive não apenas na assistência, como também em postos, como os de gerência, docência e na representação de produtos relacionados à área, o que demonstra o quanto os conhecimentos adquiridos durante a pós-graduação geraram novas possibilidades de trabalho. Deste modo, o conhecimento se torna importante e é considerado recurso indispensável no mundo globalizado e neoliberal. . $^{2,9}$

Em tal âmbito, entende-se que a universidade é uma instituição importante, visto que dispõe de instrumentos, estruturas e recursos humanos para fomentar e fortalecer a qualidade da força de trabalho. Nas universidades, viabilizam-se expressão de opiniões, atitudes e projetos que contribuem para produzir conhecimento e formar/qualificar profissionais. ${ }^{9,10}$

As universidades públicas possuem altos padrões de ensino e pesquisa, mantêm grau elevado de independência, no que concerne às determinações imediatas do mercado, apesar das pressões externas que sofrem para formar recursos 
humanos e desenvolver pesquisas, segundo o interesse do capital, e não, imperiosamente, com base em necessidades sociais. Assim, nota-se que, nesses espaços, há produção de ciência e disseminação de novos conhecimentos essenciais para a sociedade..$^{9,10}$

Isso reforça o motivo pelo qual os participantes relataram que ser especialista, egresso de universidade pública, é fator impulsionador para inserção e manutenção do estomaterapeuta no mundo do trabalho. A despeito das dificuldades que permeiam o ensino público no Brasil, ainda se tem, nesses locais, grande incentivo à produção de ciência e à qualificação de docentes, além da preocupação constante com um ensino de qualidade, em nível de graduação e pós-graduação.

O reconhecimento entre os pares também foi indicado nas entrevistas, sendo visto como elemento-chave da relação do sujeito com o trabalho e a organização laboral, que repercute diretamente nos processos motivacionais e nas percepções de valorização do trabalhador.

Assevera-se que o reconhecimento é um processo de retribuição simbólica, apontado para julgamentos sobre o fazer das pessoas. A psicodinâmica do trabalho tem correlacionado a ausência de reconhecimento aos processos de sofrimento, ao adoecimento e à despersonalização do trabalhador. ${ }^{11}$

Os egressos sinalizaram que o reconhecimento se associava ao conhecimento que esses especialistas demonstravam. A busca pelo conhecimento, notoriamente, promove novas possibilidades para os profissionais, enriquece o currículo e faz com que os trabalhadores se tornem referência nas áreas de atuação, por possuírem expertise diferenciada, concedendo-lhes oportunidades de crescimento pessoal e profissional..$^{2,12}$

Isso é fato relevante, uma vez que as pessoas desejam ser reconhecidas e valorizadas nas instituições em que atuam, constituindo fator motivador e impulsionador para o sucesso do processo de trabalho. Quando esse reconhecimento está vinculado ao conhecimento, gera no trabalhador sentimento de satisfação, fazendo com que este busque cada vez mais a qualificação. ${ }^{2,12}$

Outra questão importante a ser discutida é que o reconhecimento vai ao encontro da autonomia profissional, também apreendida nas falas dos egressos. Entende-se por autonomia a capacidade de uma pessoa e/ou grupo em fixar as próprias normas e leis, contribuindo para a independência; é concebida, então, como a capacidade de autodeterminação de um sujeito ou coletividade. Assim, a autonomia está muito relacionada à capacidade de desenvolver o processo de trabalho sem depender, necessariamente, de outros profissionais, ou dependendo o mínimo possível. ${ }^{13,14}$

A atividade autônoma obteve maior amplitude na prática profissional após o surgimento dos primeiros consultórios e clínicas de enfermagem, serviços criados com intuito de atender aos cuidados que podem ser realizados em ambiente extrahospitalar, inclusive os cuidados especializados, destacando-se os que necessitam de saber específico e, por vezes, centrados em aspectos não tão divulgados, como a estomaterapia. ${ }^{13-15}$
Enfatiza-se que, recentemente, o Conselho Federal de Enfermagem publicou a Resolução 568/2018, ${ }^{15}$ a qual versa sobre a regulamentação do funcionamento de consultórios e clínicas de enfermagem, favorecendo a ação autônoma de enfermeiros, ampliando o atendimento aos clientes de enfermagem no âmbito individual, coletivo e domiciliar, respaldando, além disso, a atuação do estomaterapeuta nesses espaços. Assim, há novas possibilidades para realização do trabalho do especialista, ensejando a independência profissional e o cuidado diferenciado, sob nova perspectiva.

Outro aspecto que emergiu das entrevistas foi o acesso às tecnologias diferenciadas para o cuidado em estomaterapia. Produtos, coberturas e equipamentos utilizados para cuidar das lesões de pele têm se diversificado e se tornado mais avançados em termos científicos e tecnológicos, o que permite um processo de cicatrização mais rápido, menos complicado e com menores danos para pacientes, quando bem indicados. ${ }^{16}$

Desse modo, existem coberturas que atuam como desbridantes, ou na prevenção de infecções, assim como produtos para higienização e antissepsia, e os que agem quando há infecção associada. Enfim, existem tecnologias arrojadas que favorecem o cuidado de excelência, desde que o profissional tenha conhecimento sobre elas e saiba indicá-las, bem como substituí-las, à medida que a situação de saúde do paciente se modifica. Destaca-se, ainda, que as tecnologias no cuidado de enfermagem estão disponíveis para as três áreas de atuação da estomaterapia, embora nem todas tenham sido citadas pelos egressos. $^{16,17}$

Por outro lado, sabe-se que a enfermagem precisa de materiais em quantidade e qualidade adequadas para prestar cuidado satisfatório. Quando isso não ocorre, há interferência prejudicial no processo de trabalho desses profissionais, já que os trabalhadores têm ciência de que poderiam utilizar produtos e equipamentos de melhor qualidade para propiciar a melhora mais rápida do paciente..$^{4,5,16,17}$

Nesse sentido, enfatiza-se que a maioria das instituições públicas vem sofrendo sucateamento profundo, o qual tem relação direta com o ideário neoliberal no setor da saúde, pois o neoliberalismo defende o enxugamento da máquina pública, portanto, há menos repasse de verbas para saúde pública. Assim, os recursos para a saúde têm diminuído, fazendo com que os profissionais da área enfrentem o grande desafio de encontrar novas medidas para coordenar os recursos escassos, de forma a direcionar a eficiência da utilização e oferecer assistência segura à população. ${ }^{3-5,8}$

Sabe-se que, de maneira geral, existe, no cenário brasileiro, principalmente nas redes públicas de saúde, dimensionamento equivocado de recursos humanos, o que fragiliza e dificulta a atuação de enfermeiros e da equipe na assistência a pacientes. Muitas instituições operam com quantitativo reduzido de profissionais de enfermagem, o que acarreta sobrecarga de trabalho e, por vezes, adoecimento no e pelo trabalho nesses indivíduos..$^{3-5,8}$

A insuficiência de enfermeiros em unidades de saúde merece destaque em pesquisas acadêmicas, estando os achados 
deste estudo em consonância com outros que sinalizam a enfermagem como uma das profissões com alto risco de estresse e adoecimento, visto que é muito prejudicada pela carência de dimensionamento adequado de recursos humanos. ${ }^{3-5,8}$

Quando se trata de recursos humanos especializados, a situação é ainda mais delicada. São raros os concursos públicos que ofertam vagas específicas para enfermeiros especialistas, pois, comumente, os cargos são para generalistas. E, como efeito do modelo neoliberal, a ocorrência de concursos públicos está cada vez mais escassa, o que compromete a reposição de vagas, resultando em não ocupação de vagas que são necessárias para o bom andamento do processo de trabalho. ${ }^{4}$

No contexto privado, não se vivencia situação muito diferente. Ainda como reflexo do neoliberalismo, surgem, cada vez mais, vagas temporárias e/ou ofertas de remuneração incompatível com a atividade a ser realizada. Isso faz com que se tenha alta rotatividade nos postos de trabalho, desfavorecendo a manutenção de profissionais em instituições. ${ }^{3-5,8}$

Apesar de a Estomaterapia ser uma especialidade relativamente nova em enfermagem, vem ganhando espaço e destaque entre os profissionais da saúde, por ser uma área de atuação em expansão entre enfermeiros. ${ }^{2,16,17}$ Além disso, devido ao aumento do número de pessoas com estomias, feridas e incontinências, a assistência a esses indivíduos vem obtendo significância a nível nacional, valorizando, deste modo, o enfermeiro estomaterapeuta, tornando-o um profissional com papel fundamental no processo de reabilitação. ${ }^{18}$

Reconhece-se, entretanto, que no Brasil não há uma cultura de valorização das especialidades de enfermagem, como em outras categorias, fator que contribui para que a estomaterapia ainda não seja tão divulgada nos serviços de saúde. 1,2,10,11 $^{1}$

Destaca-se que a Estomaterapia é uma especialidade relativamente jovem no contexto brasileiro, portanto, ainda não possui lastro histórico bem ancorado entre os profissionais da saúde e a população em geral. Tem ainda terminologia parecida com uma especialidade da odontologia - a Estomatologia - cujo objetivo é a prevenção, o diagnóstico e tratamento de doenças da boca e estruturas anexas, produzindo, consequentemente, algum grau de confusão. 2,16

Revela-se que a Estomaterapia, apesar de ser uma especialidade em expansão, ainda apresenta campo restrito para absorção de enfermeiros pós-graduados, em parte, pela carência de concursos públicos voltados essencialmente para cargos de especialistas e pelo desconhecimento de muitos profissionais da saúde e da população em geral acerca das competências e habilidades que este especialista detém. ${ }^{19}$ Como resultado, ainda se observa subutilização do estomaterapeuta no campo da promoção da saúde, prevenção de agravamento de situações, tratamento e reabilitação de pessoas com feridas, estomas e incontinência.

Muitos estomaterapeutas que atuam no mercado de trabalho acabam sendo absorvidos dentro da própria instituição, porém, frequentemente, não recebem aumento salarial após a conclusão da pós-graduação. Outros, em função da autonomia proporcionada pela estomaterapia, atuam realizando cuidados em domicílios e participando de cooperativas de trabalho que, por sua vez, não Ihes conferem direitos trabalhistas, como $13^{\circ}$ salário, auxíliodoença e férias remuneradas. Algumas dessas dificuldades, associadas à restrição dos postos de trabalho na estomaterapia, relacionam-se com o contexto neoliberal vigente. ${ }^{2}$

Nesse sentido, a configuração do mercado de trabalho de enfermeiros acompanha a lógica de redução ou inexistência de direitos trabalhistas, propiciando a mudança do padrão da composição da força de trabalho assalariada para o trabalho precário, o que provoca a diminuição das oportunidades laborais. Além disso, instaura-se o trabalho temporário como parte da estratégia de flexibilização do trabalho, resultando em insegurança no emprego e aumento da rotatividade de trabalhadores. ${ }^{3-5,8}$

\section{CONCLUSÃO E IMPLICAÇÃO PARA A PRÁTICA}

O estudo analisou as facilidades e as dificuldades vivenciadas pelos pós-graduados de um Curso de Estomaterapia, considerando a atuação como especialista no mundo do trabalho. Logo, constatou-se que as facilidades se relacionaram com o reconhecimento do especialista; a autonomia; a ascensão e o crescimento profissional; os conhecimentos adquiridos por meio da especialização; e a disponibilidade de tecnologias do cuidado para realização da prática profissional.

No que tange aos fatores dificultadores, os participantes apontaram a carência de recursos humanos, a escassez de materiais e a política institucional desfavorável. Dificuldades as quais se referem predominantemente à configuração políticoeconômica do mundo do trabalho, cujos fundamentos se pautam no ideário neoliberal, que foca na redução dos gastos do Estado, desta maneira, pouco investindo em políticas para o bem-estar social.

O presente estudo contribui para oferecer destaque e valorização a enfermeiros especialistas em Estomaterapia. Neste sentido, evidenciou-se que este profissional exerce diversas funções essenciais no cuidado a pacientes com estomias, feridas e incontinências anal e urinária. Além disso, avançouse no conhecimento, ao enumerar e discutir as facilidades e as dificuldades para inserção e manutenção deste especialista no cenário laboral, o qual é permeado pela precarização imposta pelo neoliberalismo. Ademais, apresentaram-se dados que favorecem a elaboração de estratégias e medidas para minimizar as dificuldades do exercício profissional do estomaterapeuta.

A limitação do presente estudo se situou no fato de ter investigado egressos de apenas um Curso de Especialização em Estomaterapia, no Rio de Janeiro, Brasil, o que reduz a abrangência dos resultados apreendidos, visto que se analisou uma única realidade. Futuras investigações, com maior abrangência, contemplando outras regiões nacionais são potenciais para dar seguimento aos achados deste estudo. No entanto, entende-se que esta pesquisa pode estimular a realização de investigações 
similares em outras localidades, possibilitando comparações e distanciamentos regionais.

\section{CONTRIBUIÇÕES DOS AUTORES}

Desenho do estudo. Carolina Cabral Pereira da Costa. Norma Valéria Dantas de Oliveira Souza

Coleta ou produção dos dados. Carolina Cabral Pereira da Costa. Norma Valéria Dantas de Oliveira Souza

Análise de dados. Carolina Cabral Pereira da Costa. Norma Valéria Dantas de Oliveira Souza. Samira Silva Santos Soares. Manoel Luís Cardoso Vieira. Midian Dias Oliveira. Raquel Soares Pedro. Ursula Silva Baptista Chaves.

Interpretação dos resultados. Carolina Cabral Pereira da Costa. Norma Valéria Dantas de Oliveira Souza. Samira Silva Santos Soares. Manoel Luís Cardoso Vieira. Midian Dias Oliveira. Raquel Soares Pedro. Ursula Silva Baptista Chaves.

Redação e revisão crítica do manuscrito. Samira Silva Santos Soares. Carolina Cabral Pereira da Costa. Manoel Luís Cardoso Vieira. Midian Dias Oliveira. Raquel Soares Pedro. Ursula Silva Baptista Chaves. Norma Valéria Dantas de Oliveira Souza

Aprovação da versão final do artigo. Samira Silva Santos Soares. Carolina Cabral Pereira da Costa. Manoel Luís Cardoso Vieira. Midian Dias Oliveira. Raquel Soares Pedro. Ursula Silva Baptista Chaves. Norma Valéria Dantas de Oliveira Souza

Responsabilidade por todos os aspectos do conteúdo e a integridade do artigo publicado. Samira Silva Santos Soares. Carolina Cabral Pereira da Costa. Manoel Luís Cardoso Vieira. Midian Dias Oliveira. Raquel Soares Pedro. Ursula Silva Baptista Chaves. Norma Valéria Dantas de Oliveira Souza

\section{EDITOR ASSOCIADO}

Cristina Rosa Baixinho

\section{REFERÊNCIAS}

1. Silva RMO, Luz MDA, Fernandes JD, Silva LS, Cordeiro ALAO, Mota LSR. Becoming a specialist: Portuguese nurses' expectations after completing the specialization program. Rev Enf Ref. 2018;4(16):147-54 http://dx.doi.org/10.12707/RIV17076.

2. Gontijo TG, Borges EL, Ferraz AF, Pires Jr JF, Spira JAO. Atuação profissional dos estomaterapeutas egressos da Universidade Federal de Minas Gerais. Estima. 2019;17:1-11. http://dx.doi.org/10.30886/ estima.v17.686_PT.

3. Machado MH, Oliveira E, Lemos W, Lacerda WF, Aguiar Filho W, Wermelinger $\mathrm{M}$ et al. Mercado de trabalho da Enfermagem: aspectos gerais. Enferm Foco. 2015;6(1-4):43-78. http://dx.doi.org/10.21675/2357707X.2016.v7.nESP.

4. Souza NVDO, Gonçalves FGA, Pires AS, David HMSL. Neoliberalist influences on nursing hospital work process and organization. Rev Bras
Enferm. 2017;70(5):961-9. http://dx.doi.org/10.1590/0034-7167-20160092. PMid:28977215.

5. Gonçalves FGA, Souza NVDO, Zeitoune RCG, Adame GFPL, Nascimento SMP. Impacts of neoliberalism on hospital nursing work. Texto Contexto Enferm. 2015;24(3):646-53. http://dx.doi.org/10.1590/010407072015000420014.

6. D'Avila TB. Satisfação no trabalho de enfermeiros recém egressos [dissertação]. Rio de Janeiro: Universidade Federal do Estado do Rio de Janeiro; 2012.

7. Bardin L. Análise de Conteúdo. Tradução: Luís Augusto Pinheiro. São Paulo: Edições 70; 2016.

8. Marques DO, Pereira MS, Souza ACS, Vila VSC, Almeida CCOF, Oliveira EC. Absenteeism - illnes of the nursing staff os a universyt hospital. Rev Bras Enferm. 2015;68(5):594-600. http://dx.doi.org/10.1590/00347167.2015680516i

9. Püschel VAA, Costa D, Reis PP, Oliveira LB, Carbogim FC. Nurses in the labor market: professional insertion, competencies and skills. Rev Bras Enferm. 2017 dez;70(6):1220-6. http://dx.doi.org/10.1590/00347167-2016-0061. PMid:29160483.

10. Lusa MG, Martinelli T, Moraes AS, Almeida TP. The public university in times of neoliberal adjustment and loss of rights. Rev Katálysis. 2019;22(3). http://dx.doi.org/10.1590/1982-02592019v22n3p536.

11. Dejours C. Trabalho vivo: trabalho e emancipação. Brasília: Paralelo;2016.

12. Silva EM, Moreira MCN. Health team: negotiations and limits of autonomy, belonging and the acknowledgement of others. Cien Saude Colet. 2015;20(10):3033-42. http://dx.doi.org/10.1590/1413812320152010.20622014. PMid:26465846.

13. Santos Él, Oliveira JGAD, Ramos RS, Silva ACSS, Belém LS, Silva AL Facilidades e dificuldades à autonomia profissional de enfermeiros no cuidado de pessoas com feridas: estudo de representações sociais. Estima. 2017;15(1):3-9. http://dx.doi.org/10.5327/Z1806-3144201700010002.

14. Santos Él, Alves YR, Silva ACSS, Gomes AMT. Professional autonomy and nursing: representations of health professionals. Rev Gaúcha Enferm. 2017;38(1):e59033. http://dx.doi.org/10.1590/1983-1447.2017.01.59033. PMid:28538807.

15. Resolução $\mathrm{n}^{\circ} \mathbf{5 6 8}$ de 9 de dezembro de 2018 (BR). Aprova o regulamento dos consultórios de enfermagem e clínicas de enfermagem. Conselho Federal de Enfermagem [periódico na internet] 2018 [cited 27 may 2020]. Available from: http://www.cofen.gov.br/resolucao-cofenno-0568-2018 60473.htm

16. Shoji S, Souza NVDO, Maurício VC, Costa CCP, Alves FT. O cuidado de enfermagem em Estomaterapia e o uso das tecnologias. Estima. 2017;15(3):169-77. http://dx.doi.org/10.5327/Z1806-3144201700030008.

17. Tavares WS, Silva RS. Curativos utilizados no tratamento de queimaduras: uma revisão integrativa. Rev Bras Queimaduras [Internet] 2015 [cited 2020 May 22];14(4):300-6. Available from: http://www.rbqueimaduras. com.br/details/282/pt-BR/curativos-utilizados-no-tratamento-dequeimaduras--uma-revisao-integrativa.

18. Miranda LSG, Carvalho AAS, Paz EPA. Quality of life of ostomized person: relationship with the care provided in stomatherapy nursing consultation. Esc Anna Nery. 2018;22(4):e20180075. http://dx.doi. org/10.1590/2177-9465-ean-2018-0075

19. Avila LI, Silveira RS, Lunardi VL, Fernandes GFM, Mancia JR, Silveira JT. Implications of the visibility of professional nursing practices. Rev Gaúcha Enferm. 2013;34(3):102-9. http://dx.doi.org/10.1590/S198314472013000300013. PMid:24344591. 